\title{
Cambios en la Textura y Color en Mango (Tommy Atkins) Pre- Secado por Deshidratación Osmótica y Microondas
}

\author{
Armando Alvis-Bermudez ${ }^{(1)}$, Carlos García-Mogollon ${ }^{(2)^{*}}$ y Saúl Dussán-Sarria(3) \\ (1) Facultad de Ingenierías, Univ. de Córdoba, Programa de Ing. de Alimentos, Grupo de Investigación \\ Procesos y Agroindustria de Vegetales, Carrera $6 \mathrm{~N}^{\circ} 76-103, \mathrm{Km} 3$, vía Cereté. Córdoba - Colombia. \\ (2) Facultad de Ingeniería, Universidad de Sucre. Sincelejo, Sucre, Colombia. \\ (e-mail: carlos.garcia@unisucre.edu.co). \\ (3) Facultad de Ingeniería y Administración, Depto. de Ingeniería, Univ. Nacional de Colombia. \\ Sede Palmira, Palmira, Valle del Cauca, Colombia. \\ * Autor a quien debe ser dirigida la correspondencia.
}

Recibido Sep. 1, 2015; Aceptado Nov. 6, 2015; Versión final Dic. 23, 2015, Publicado Abr. 2016

\begin{abstract}
Resumen
En el presente trabajo, se evaluó el efecto de la deshidratación osmótica (65 ${ }^{\circ}$ Brix de 37 a $40{ }^{\circ} \mathrm{C}$ por 60 $\mathrm{min}$ ), microondas (560 W por $7 \mathrm{~min}$ ) y combinado como pre-secado de mangos. Los mangos con 11 a 14 ${ }^{\circ}$ Brix y humedad del $80 \%$ fueron dimensionados a $1 \times 1 \times 0.4 \mathrm{~cm}$ y deshidratados a $70{ }^{\circ} \mathrm{C}$ en un horno convectivo y al sol. Se evaluó el croma, la tonalidad y los parámetros de textura. Se utilizó un diseño con arreglo unifactorial correspondiente a la variable categórica deshidratación. Los parámetros de color y textura presentaron diferencias significativas $(p<0.05)$. El tratamiento de deshidratación más adecuado que le confiere a los trozos de mango el mantenimiento del color, textura firme, mejor aceptación sensorial y menor tiempo de secado es el de deshidratación osmótica seguido del tratamiento convencional utilizando convección forzada de aire.
\end{abstract}

Palabras clave: ; mango; Mangifera Indica; deshidratación; firmeza; color; textura.

\section{Changes in the Texture and Color in Mango (Tommy Atkins) Pre-Drying by Osmotic Dehydration and Microwave}

\begin{abstract}
In the present work, the effect of osmotic dehydration ( $65^{\circ} \mathrm{Brix}$ of 37 to $40^{\circ} \mathrm{C}$ for $60 \mathrm{~min}$ ), microwave (560 $\mathrm{W}$ by $7 \mathrm{~min}$ ) and combined as pre-drying of mangos, was evaluated. The mangos with 11 to $14{ }^{\circ} \mathrm{Brix}$ and $80 \%$ humidity were rated to $1 \times 1 \times 0.4 \mathrm{~cm}$ and dehydrated at $70^{\circ} \mathrm{C}$ in a convective oven and under the sun. Characteristics such as Chroma, hue and texture parameters were determined. A univariate design, corresponding to the categorical variable dehydration, was employed. The parameters of color and texture presented significant differences $(p<0.05)$. The most appropriate treatment of dehydration which maintained features of appearance of the flakes of mango was drying by osmotic dehydration followed by conventional treatment using air forced convection.
\end{abstract}

Keywords: mango; Mangifera Indica; dehydration; firmness; color; texture 


\section{INTRODUCCIÓN}

El mango (Mangifera indica L.) es una fruta tropical caracterizada por su valor nutricional, color, sabor y composición en fibra que se manifiesta en la percepción fibrosa que se tiene al consumir. Sin embargo, las pérdidas poscosecha en el manejo y procesamiento hacen necesario aplicar tecnologías de conservación que conduzcan al desarrollo de nuevos productos procesados que mantengan o mejoren el valor sensorial y nutricional de esta fruta (Zapata y Montoya 2012; Varela et al., 2013; Zou et al., 2013). Las tendencias de consumo saludable colocan a las frutas y vegetales en un primer nivel de necesidad, sin dejar de lado los atributos sensoriales visuales y táctiles cuantificados por la medida del color y la textura. El primero expresado como una magnitud psicofísica y el segundo como una manifestación funcional de la estructura, mecánica y propiedades superficiales del alimento detectadas a través de los sentidos de la visión, oído, tacto y el sabor (Zuluaga, 2012).

Tecnologías como la cocción en aceite caliente (Torezan et al., 2007), fritura al vacío (Nunes y Moreira, 2009), liofilización (Wang et al., 2010; Sogi et al., 2015), puffing (He et al., 2011) han sido sujetas a estudios presentando factores positivos asociados a la retención del color, sabor y textura atractiva para el consumidor (Zou et al., 2013). Siendo estas dos últimas una metodología de secado que produce alimentos secos de alta calidad. Una alternativa en la eliminación de humedad es aplicando la deshidratación osmótica (DO) o la tecnología emergente por microondas; la primera es un método no térmico de deshidratación mediante el cual la fruta se sumerge en una solución hipertónica dándose procesos de transferencia de agua, de soluto y solutos nutrientes (Reppa et al., 1999). El microondas como tecnología no convencional donde el calentamiento se da del centro del producto a la superficie genera que el agua caliente migre a la superficie y se produzca el secado.

Zuluaga et al. (2010) estudiaron la combinación de tecnologías aplicando DO como pretratamiento al secado convencional donde evaluaron la influencia del proceso y la temperatura de secado $\left(60{ }^{\circ} \mathrm{C} \mathrm{y} 70{ }^{\circ} \mathrm{C}\right)$ sobre las características físicas del mango (Tommy Atkins), Della y Mascheroni (2011) en papa, Garcia et al. (2013) en piña, Ochoa et al. (2012) evaluaron las características de secado de rebanadas de mango en ventana refractiva con temperatura del agua a $92^{\circ} \mathrm{C}$, Villalpando et al. (2011) aplicaron microondas (1000 W) de tres diferentes rebanadas de mango (ovalado, longitudinal y transversal) como complemento al secado de muestras osmodeshidratadas. La pérdida de peso, tiempo de secado, cambios de color y preferencia de los consumidores son factores que se afectan por la combinación de deshidratación osmótica, microondas y combinación (DO y MW) previa al secado en estufa y solar. El objetivo de esta investigación fue evaluar los cambios en la textura y el color de trozos de mango (Tommy Atkins) deshidratados por secado convencional cuando sometidos a tratamientos previos de deshidratación osmótica y por microondas.

\section{MATERIALES Y MÉTODOS}

\section{Obtención y adecuación de la materia prima}

Los mangos (Tommy Atkins) utilizados como materia prima se seleccionaron con un índice de madurez de 0,125 , de $11^{\circ}$ Brix a $14^{\circ}$ Brix y humedad $80 \pm 1 \%$ los cuales se sometieron a operaciones de lavado, pelado, troceado para obtener trozos de una dimensión de $1 \mathrm{~cm} \times 1 \mathrm{~cm} \times 0,4 \mathrm{~cm}$. En la determinación de los sólidos solubles se utilizó una lectura refractométrica (AOAC 932.12/90). La acidez se midió por el método de titulación potenciométrica (AOAC 942.05/90). El índice de madurez se calculó empleando la relación de sólidos solubles/ acidez.

\section{Deshidratación de trozos de mango}

La deshidratación de los trozos de mango se realizó en dos etapas consecutivas pre-secado y secado. En la primera etapa se aplicó la tecnología de deshidratación osmótica (DO) con una solución de sacarosa de $65^{\circ}$ Brix por 60 min y temperatura ambiente de 37 a $40^{\circ} \mathrm{C}$ en una relación peso muestra:volumen solución osmótica 1:5, condiciones experimentales similares fueron usadas por Zou et al. (2013), en seguida la deshidratación en microondas (MW) domestico de marca Haceb HM-1.1 con una frecuencia de $2450 \mathrm{MHz}$ y dimensiones internas de $354 \mathrm{~mm}$ x $228 \mathrm{~mm}$ x $373 \mathrm{~mm}$. Se tomaron $50 \mathrm{~g}$ de muestra y se distribuyeron uniformemente en el plato del microondas a una potencia de $560 \mathrm{~W}$ por $7 \mathrm{~min}$ y la combinación de estas (DO+MW). En la segunda etapa se aplicaron los métodos de deshidratación aquí denominados convencionales: el secado al sol en bandejas de $50 \mathrm{~g}$ de muestra y se expusieron a la radiación solar de 9:00 a las 15:00 y el método convencional utilizando un horno de convección forzada marca Technicook modelo MINICONV con dimensiones $580 \mathrm{~mm}$ de ancho, $630 \mathrm{~mm}$ de profundidad, $435 \mathrm{~mm}$ de alto a una temperatura de $70^{\circ} \mathrm{C}$ (AOAC 925.09). El contenido de agua final de los trozos de mango fue fijado en $10 \%$. La experimentación se realizó en la Universidad de Córdoba, Colombia, Sede Berastegui. 


\section{Contenido de agua}

Se determinó el contenido de agua de las muestras cada 10 min las primeras $2 \mathrm{~h}$ de proceso y luego cada 30 min hasta peso constante. El contenido de agua se expresó bajo la relación el peso húmedo y peso del sólido seco (MS) a un determinado tiempo (g agua /g MS). La masa de las muestras durante el secado se obtuvo pesando en una balanza analítica (MettlerPS4000 con precisión de $\pm 0,01 \mathrm{~g}$ ).

\section{Color}

El análisis de color se realizó determinando las coordenadas de color del espacio CIELAB ( $\mathrm{L}^{*}$ : luminosidad o claridad; $\mathrm{a}^{*}$ : coordenada rojo-verde y $\mathrm{b}^{*}$ : coordenada amarillo-azul). Las mediciones se hicieron con un colorímetro Colorflex EZ 45 (HunterLab®). El colorímetro se calibró con un plato de cerámica estándar de color verde y blanco estándar antes de su lectura. A partir de estos parámetros de color se calcularon las magnitudes psicofísicas croma $\left(\mathrm{C}^{*} \mathrm{ab}\right)$ y tono $\left(\mathrm{H}^{*} \mathrm{ab}\right)$ utilizando las siguientes ecuaciones:

$$
\begin{aligned}
& C^{*}{ }_{a b}=\sqrt{a^{*} 2+b^{* 2}} \\
& H^{*}{ }_{a b}=\arctan \frac{b^{*}}{a^{*}}
\end{aligned}
$$

\section{Textura}

La fuerza de fractura se midió en un analizador de textura (TA-XT2i, Stable Micro System, UK), con un accesorio de penetración cilíndrico de $2 \mathrm{~mm}$ de diámetro (SMSP/2) y el software Texture Expert Exceed versión 2.64. Las pruebas fueron sometidas a penetración unidireccional a una velocidad de $2 \mathrm{~mm} / \mathrm{s}$. La fuerza de fractura se definió como el punto donde se produce una caída apreciable de la fuerza causada por el rompimiento parcial o total de la muestra (Ciro-Velásquez et al., 2007). Por cada muestra evaluada se realizaron 15 pruebas.

\section{Evaluación sensorial}

Las muestras de mango deshidratado se evaluaron mediante un panel sensorial integrado por 50 consumidores de 20 a 25 años, utilizando una prueba hedónica de ordenamiento con 8 puntos siendo 1 la de menor preferencia y 8 la de mayor preferencia. Se realizaron la agrupación de consumidores mediante la Clasificación Jerárquica Ascendente (CJA) para la formación de clases o segmentos. Se usó el Análisis de Componentes Principales (ACP) para la correlación de los datos instrumentales-sensoriales..

\section{Diseño experimental}

Se aplicó un diseño completamente al azar con arreglo unifactorial correspondiente a la variable categórica Deshidratación con ocho tratamientos (Tabla 1) y 3 repeticiones para un total de 24 unidades experimentales. Para determinar entre que niveles del factor fueron significativas las diferencias se aplicó el Test de Tukey con un nivel de significancia de $p<0.05$ y se evaluaron por la matriz de correlación Pearson. Para el análisis de los datos experimentales se utilizó el programa estadístico Statgraphics Centurion XV el cual contaba con licencia de uso por parte de la Universidad de Córdoba.

Tabla 1. Matriz del diseño experimental

\begin{tabular}{|c|c|c|c|}
\hline Tratamiento & Pre-secado & Deshidratación & Tiempo secado (min) hasta contenido de agua \\
\hline T1 & STP & Sol & $237.0 \pm 4.6$ \\
\hline T2 & STP & Horno convectivo & $75.0 \pm 1.0$ \\
\hline T3 & Deshidratación Osmótica & Sol & $100.0 \pm 5.7$ \\
\hline T4 & Deshidratación Osmótica & Horno convectivo & $51.6 \pm 7.6$ \\
\hline T5 & Microondas & Sol & $148.6 \pm 8.0$ \\
\hline T6 & Microondas & Horno convectivo & $28.6 \pm 1.2$ \\
\hline T7 & Deshidratación Osmótica + Microondas & Sol & $43.3 \pm 5.7$ \\
\hline T8 & Deshidratación Osmótica + Microondas & Horno convectivo & $24 \pm 1.7$ \\
\hline
\end{tabular}

STP: Sin Tratamiento Previo 


\section{RESULTADOS Y DISCUSIÓN}

\section{Caracterización fisicoquímica de trozos de mango}

Las propiedades físico-químicas iniciales del mango usado fueron $12.3 \pm 0.7{ }^{\circ}$ Brix, $0.69 \pm 0.1 \%$ acidez expresada como ácido cítrico, 58,16 croma $\left(\mathrm{C}^{*}\right.$ ab) y 1,37 de tonalidad $\left(\mathrm{H}^{*}{ }^{*} \mathrm{~b}\right), 80 \%$ de humedad y grado 4 de madurez de acuerdo a norma ICONTEC (2003) para la variedad Tommy Atkins.

\section{Caracterización del color}

En la caracterización colorimétrica de trozos de mango deshidratados las magnitudes psicofísicas croma $\left(\mathrm{C}^{*}{ }_{a b}\right)$, tono $\left(\mathrm{H}^{*}{ }_{a b}\right)$ mostraron diferencias estadísticas significativas $(\mathrm{p}<0.05)$ y se identificaron 3 grupos estadísticamente homogéneos en la prueba de comparación de tratamientos entre medias utilizando la prueba Tukey (Tabla 2). El efecto de la deshidratación sobre el color de trozos de mango es observable a través del amarillo característico del mango maduro que presenta diferentes tonalidades que lo hacen más o menos atractivo. Los tratamientos sometidos a la DO en general adquieren un brillo y solidez en el color amarillo, mientras que las tratadas al MW son más opacas. Lo cual indica que el pre-secado influyó en los cambios de color de trozos, siendo el más adecuado el secado previo por DO. Cuanto más largo sea el proceso de deshidratación y más elevada la temperatura, mayores son las pérdidas en los pigmentos en los alimentos (Rahman y Perera, 1999; Lee y Schwartz 2006). Seguramente la solidez del color amarillo de los trozos de mango se debió al no uso de temperaturas mayores a $40^{\circ} \mathrm{C}$.

Tabla 2. Valores de Croma y Tono de trozos de mango deshidratado

\begin{tabular}{|c|c|c|}
\hline Tratamiento & $\left(\mathrm{C}^{*} \mathrm{ab}\right)$ & $\left(\mathrm{H}^{*} \mathrm{ab}\right)$ \\
\hline T4 & $49.88 \mathrm{a}$ & $1.33 \mathrm{ab}$ \\
\hline T8 & $48.41 \mathrm{ab}^{*}$ & $1.32 \mathrm{ab}{ }^{\star}$ \\
\hline T7 & $46.78 \mathrm{ab}^{*}$ & $1.33 \mathrm{ab}$ \\
\hline T3 & $45.46 \mathrm{abc}{ }^{*}$ & $1.36 \mathrm{a}$ \\
\hline T6 & $49.19 \mathrm{a}^{*}$ & $1.33 \mathrm{ab}$ \\
\hline T5 & $37.93 \mathrm{c}^{\star}$ & $1.27 \mathrm{c}^{*}$ \\
\hline T2 & $50.86 \mathrm{a}$ & $1.31 \mathrm{bc}^{*}$ \\
\hline T1 & $40.86 \mathrm{bc}^{*}$ & $1.32 \mathrm{ab}$ \\
\hline
\end{tabular}

Los caracteres derivados Cab y Hab indican la intensidad del color y el color verdadero, respectivamente (Salinas et al., 2010). Así, la disminución de Cab indicó una menor intensidad del color amarillo característico del mango fresco y la disminución en Hab una variación del color hacia un amarillo más claro. El proceso de deshidratación microondas más secado al sol (T5) redujo en mayor grado las características del mango deshidratado respecto al mango fresco. Dada las características estructurales que VásquezCaicedo et al. (2006) observaron sobre la presencia de carotenoides en el mesocarpio del cv. 'Tommy Atkins', depositados en forma de plastoglóbulos dentro de cromoplastos globulares y retículo-tubulares y algunos parcialmente solubilizados en gotas lipídicas, demuestran que el pre-secado osmótico favorece la retención de los pigmentos por formación de la corteza de jarabe y la caramelización en la superficie de la hojuela de mango. Estas mismas evidencias se observaron en esta experimentación se observó brillo y solidez en el color amarillo cuando se utiliza la osmodeshidratación.

La concentración de los agentes osmóticos es un factor que afecta el proceso (Phisut et al., 2013) y se han observado diferencias en el color y otros atributos sensoriales comparando mango (Tommy Atkins) fresco e impregnado al vacío con sales de calcio (Ostos et al., 2012). Los cambios en los parámetros de color son debidos principalmente al índice de madurez, las condiciones de proceso y las características físicas. El efecto del proceso de deshidratación en la superficie del mango se ve reflejado por los cambios y pérdida de color (Gómez, 2013; Chacón y Esquivel, 2013) por oxidación extensiva provocando una pérdida en carotenoides que es incrementada por la prolongación de la deshidratación (Zuluaga et al., 2010). La deshidratación puede incrementar la concentración de carotenoides (Nimmanpipug y Therdthai, 2013; Phisut et al., 2013) causando un efecto contrario en el color del producto final.

\section{Textura}

El análisis de ANOVA determinaron diferencias significativas $(p<0.05)$ entre el parámetro textural Firmeza $(N)$ de los trozos de mango deshidratadas, identificándose 6 grupos homogéneos mediante la prueba de 
comparación de medias de Tukey. Para el parámetro Consistencia y Trabajo (N.mm) $(p<0.05)$ se identificaron 5 grupos homogéneos. El comportamiento de la textura en los diferentes procesos de deshidratación se puede observar en la Tabla 3. El mayor valor de la firmeza corresponde a la muestra deshidratada al sol, y hay una correlación positiva (1.00) de los parámetros Consistencia y Trabajo; mientras que para la Firmeza la correlación con los otros dos es de 0.895 . Es importante destacar que las mediciones de los parámetros texturales presentaron los coeficientes de variación de $44.2 \pm 0.13 \%$ en Firmeza, $42.3 \pm 0.15 \%$ Consistencia y Trabajo $2.7 \pm 0.015 \%$, esto se debe posiblemente a la falta de uniformidad en cuanto a superficie.

En consecuencia, la diferencia de mecanismos se refleja en las propiedades sensoriales de los trozos de fruta después del secado previo, evidenciándose que los osmodeshidratadas tienen mayor brillo y estructura que las tratadas por microondas debido a la ganancia de sólidos y el medio de solución azucarada en que se produce; además, los trozos tratados con microondas se observan más aplanadas producto de que la estructura de la matriz de mango no es rígida y por lo tanto no compensa la salida del agua desde el interior. Cuando se combinaron los secados previos iniciando con la osmodeshidratación se redujeron los efectos negativos del microondas. Zuluaga et al. (2010) estudiaron la combinación de tecnologías, aplicando DO como pretratamiento al secado convencional confirmando que el secado de mango sin pretratamiento a $70^{\circ} \mathrm{C}$ presento la mayor fuerza de fractura y los pretratamientos con deshidratación osmótica presentaron una menor fuerza de fractura.

Tabla 3. Parámetros de textura de trozos de mango deshidratado

\begin{tabular}{|c|c|c|c|}
\hline Tratamiento & Firmeza $(\mathrm{N})$ & Consistencia(N. s) & Trabajo (N.mm) \\
\hline T4 & $0.042 \pm 0.017 \mathrm{a}$ & $0.076 \pm 0.029 \mathrm{a}$ & $0.744 \pm 0.007 \mathrm{a}$ \\
\hline T8 & $0.059 \pm 0.035 \mathrm{~b}$ & $0.131 \pm 0.071 \mathrm{~b}$ & $1.279 \pm 0.075 \mathrm{~b}$ \\
\hline T7 & $0.065 \pm 0.037 \mathrm{~b}$ & $0.149 \pm 0.097 \mathrm{c}$ & $1.455 \pm 0.016 \mathrm{c}$ \\
\hline T3 & $0.025 \pm 0.007 \mathrm{c}$ & $0.064 \pm 0.021 \mathrm{a}$ & $0.630 \pm 0.035 \mathrm{a}$ \\
\hline T6 & $0.063 \pm 0.025 \mathrm{~b}$ & $0.117 \pm 0.039 \mathrm{~d}$ & $1.140 \pm 0.023 \mathrm{~d}$ \\
\hline T5 & $0.081 \pm 0.047 \mathrm{~d}$ & $0.153 \pm 0.084 \mathrm{c}$ & $1.500 \pm 0.066 \mathrm{c}$ \\
\hline T2 & $0.051 \pm 0.021 \mathrm{e}$ & $0.093 \pm 0.037 \mathrm{e}$ & $0.913 \pm 0.032 \mathrm{e}$ \\
\hline T1 & $0.090 \pm 0.026 \mathrm{f}$ & $0.41 \mathrm{~b} \pm 0.024 \mathrm{c}$ & $1.380 \pm 0.043 \mathrm{bc}$ \\
\hline
\end{tabular}

\section{Análisis de componentes principales}

El ACP muestra en el círculo de correlación (Figura 1) que los dos ejes principales se encontraron con el $88,22 \%$ de la variación total de los datos, observando que los atributos de textura firmeza y consistencia contribuyeron en la formación de F1; mientras los atributos de color $\left(\mathrm{C}^{*}\right)$ lo hacen para F2. La deshidratación al sol sin secado previo (T1), secado previo con microondas (T5), DO (T3) y DO-convectivo (T4) contribuyen con F1 y el resto contribuye principalmente con F2. La correlación de Pearson muestra que los parámetros texturales firmeza, consistencia y trabajo de corte es positiva $(p<0.05)$, color $\left(C^{*}\right)$ y $\left(H^{*}\right)$ es positiva $(0,449$, $\mathrm{p}<0.05)$ y mientras que la correlación es negativa entre los parámetros de textura y color.

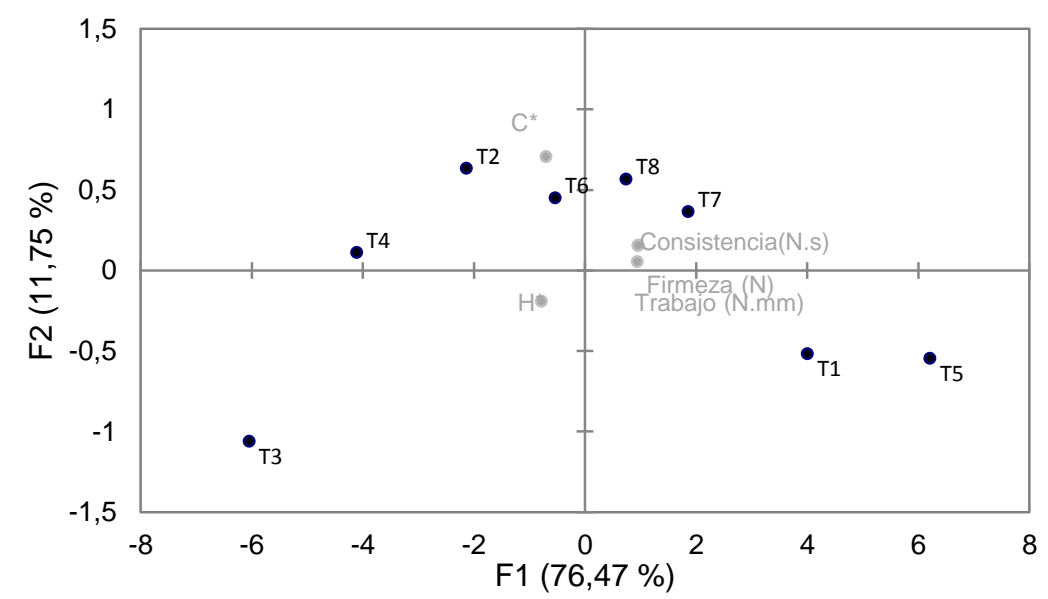

Fig. 1: Espacio físico-químico y tratamientos de trozos de mango deshidratado 


\section{Evaluación sensorial}

El análisis de ANOVA determinaron diferencias significativas $(p<0.05)$ de las formulaciones respecto a las respuestas hedónicas en la aceptación general. Se identificaron 4 segmentos de consumidores (Figura 2) mediante la aplicación de la Clasificación Ascendente Jerárquica para los datos de aceptación general. El segmento 1 se conformó con 27 de consumidores, el segmento 2 con 12, el segmento 3 con 2 y el segmento 4 con 9 consumidores.

Los consumidores del segmento 3 no tuvieron un efecto significativo y no explican la alta preferencia de este grupo de consumidores (2) por el proceso T1 (secado al sol). El número de consumidores del segmento 1, 2 y 4 resulta más representativo en explicar su preferencia por T3 y T4 caracterizados por la deshidratación osmótica como presecado con notas entre 6.44 y 7.66. Ramírez et al. (2010) obtuvieron resultados cuando aplicaron los modelos vectoriales y circulares en el análisis de componentes principales en investigación para explicar mejor la preferencia de los consumidores.

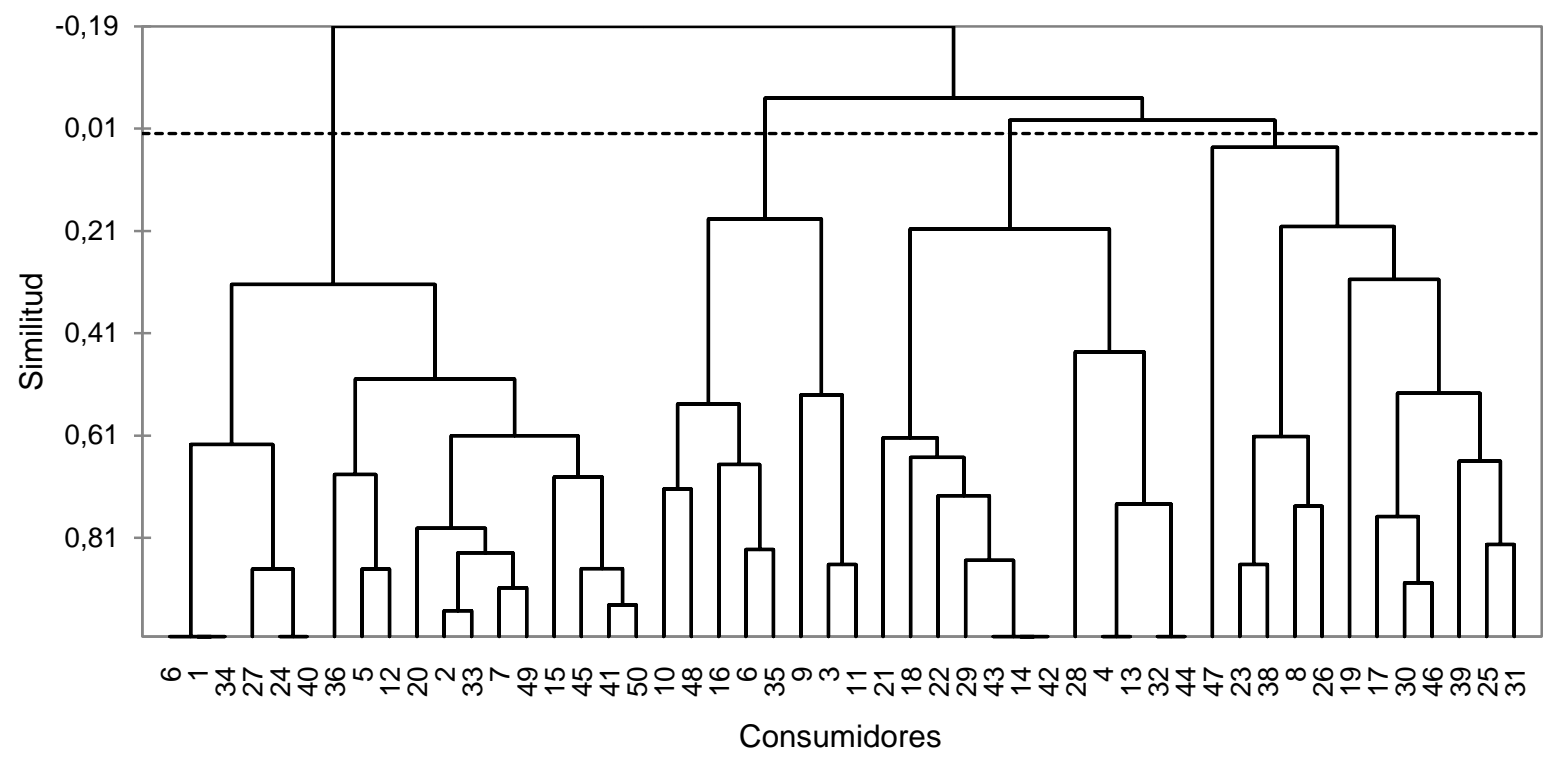

Fig. 2: Agrupaciones de consumidores según evaluación sensorial de trozos de mango deshidratado

\section{CONCLUSIONES}

Los parámetros de color de los trozos de mango (Tommy Atkins) se demeritaron por el proceso de deshidratación con excepción cuando se aplica deshidratación osmótica.

El presecado con microondas altera en mayor proporción los parámetros de textura, consistencia y trabajo de corte, que al combinarse con el secado al sol resulta en trozos más rígidos. Mientras la deshidratación osmótica dado el depósito de sólidos y la caramelización hacen la superficie de los trozos menos firmes y más elásticos.

El tratamiento de deshidratación más adecuado que le confiere a los trozos de mango el mantenimiento del color, textura firme, mejor aceptación sensorial y menor tiempo de secado es el de deshidratación osmótica seguido del tratamiento convencional utilizando convección forzada de aire.

\section{REFERENCIAS}

A.O.A.C.: Association of Official Analytical Chemist. Official Methods of Analysis, Section 954.10. 13th. Ed. Washington D.C., USA (1990)

Chacón, T. y P. Esquivel, Frutos tropicales como fuente de carotenoides: biosíntesis, composición, biodisponibilidad y efectos del procesamiento, Rev. Venez. Cienc. Tecnol. Aliment, 4 (1), 001-023 (2013)

Ciro-Velásquez, H.J., O.H. Buitrago-Giraldo y S.A. Pérez Arango, Estudio preliminar de la resistencia mecánica a la fractura y fuerza de firmeza para fruta de uchuva (Physalis peruviana L.). Revista de la Facultad Nacional de Agronomía, 60(1), 3785-3796 (2007) 
Della, P. y R. Mascheroni, Deshidratación de papas por el método combinado de secado: deshidratación osmótica, secado por microondas y convección por aire caliente, Revista Proyecciones, 9(2), 12-25 (2011)

García, A., S. Muñiz, A. Hernández, L. González, y D. Fernández, Análisis comparativo de la cinética de deshidratación Osmótica y por Flujo de Aire Caliente de la Piña (Ananas Comosus, variedad Cayena lisa), Revista Ciencias Técnicas Agropecuarias, 22(1), 62-69 (2013)

Gómez, R, Evaluación sensorial de láminas de mango (Manguifera indica L. cv. Keitt) fortificadas con cloruro de calcio mediante deshidratación osmótica con pulsos de vacío. Rev. Venez. Cienc. Tecnol. Aliment., 4 (2), 157-169 (2013)

He, X. Y., J.F. Liu y Z.H. Huang, Preparation of cold brew tea by explosión puffing drying at variable temperature and pressure. Drying Technology, 29(8), 888-895 (2011)

ICONTEC, Norma Técnica Colombiana 5210. Frustas frescas. Mango variedades mejoradas. Especificaciones. (2003)

Lee, J.H. y S.J. Schwartz, Pigmensts in plant foods, in Handbook of science, technology and engineering by Y.J. Hui, pp 14.1-14.13 Taylor \& Francis, Boca Raton, FL (2006)

Nimmanpipug, N. y N. Therdthai, Effect of osmotic dehydration time on hot air drying and microwave vacuum drying of papaya, Food and Applied Bioscience J., 1(1), 1-10 (2013)

Nunes, Y. y R.G. Moreira, Effect of osmotic dehydration and vacuum-frying parameters to produce high-quality mango chips, J. Food Science, 74(7), 355-362 (2009)

Ochoa, C., P. Quintero, A. Ayala y M. Ortiz, Drying characteristics of mango slices using the Refractance Window Technique, J. Food Engineering, 109: 69-75 (2012)

Ostos-A., S., A. Díaz y H. Suárez, Evaluación de diferentes condiciones de proceso en la fortificación de mango (Tommy Atkins) con calcio mediante impregnación a vacío, Revista Chilena de Nutrición, 39(2), 181$190(2012)$

Phisut, N., M. Rattanawedee y K. Aekkasak, Effect of osmotic dehydration process on the physical, chemical and sensory properties of osmo-dried cantaloupe, International Food Research J., 20(1), 189-196 (2013)

Rahman, M.S. y C.O. Perera, Drying and food preservation, in Handbook of food preservation by M.S. Rahman, pp 173-216 Marcel Dekker, New York, NY (1999)

Ramírez, E., L. Ramón, A. Shain, Y. González, J. Juárez, C. Martínez, H. Bravo y J. Rodríguez, Mapa externo de preferencias con datos sensoriales e instrumentales para la evaluación de salchichas de Euthynnus lineatus, Temas de Ciencia y Tecnología, 14(42), 19-28 (2010)

Reppa, A., J. Mandala, A.K. Kostaropoulos y G.D. Saravacos, Influence of solute temperature and concentration on the combined osmotic and air drying, Drying Technology, 17(7-8), 1449-1458 (1999)

Salinas, R., M. Pirovani, A. Gardea y G. González, Cambios fisicoquímicos y sensoriales limitantes de la vida de anaquel de mango fresco cortado, Rev. Fitotec. Mex. Vol. 33 (3), 215 - 223 (2010)

Sogi, D., S. Muhammad y D. Kirk, Total phenolics, carotenoids and antioxidant properties of Tommy Atkin mango cubes as affected by drying techniques, doi: 10.1016/j.Iwt.2014.04.015, LWT-Food Science and Technology. Vol. 62 (1), 564-568 (2015)

Torezan, G.A.P., H.C. Menezes, M.E. Katekawa y M.A. Silva, Microstructure and adsorption characteristics of mango chips obtained by osmotic dehydration and deep fat frying, Drying Technology, 25(1), 153-159 (2007)

Varela, H., F. Viluzca y S. Betzabé, Actividad antioxidante, análisis sensorial y microbiólogico de láminas flexibles de mango (Mangifera indica I.). Scientia Agroalimentaria, 1, 26-32 (2013)

Vásquez-Caicedo, A., A. Heller, S. Neidhart y C. Reinhold, Chromoplast morphology and $\beta$-carotene accumulation during postharvest ripening of mango cv. 'Tommy Atkins', J. Agricultural and Food Chemistry, 54(16), 5769-5776 (2006)

Villalpando, J., E. Herrera, L. Amaya-Delgado, M. Godoy, J. Mateos y S. Rodríguez, Effect of Complementary Microwave Drying on Three Shapes of Mango Slices, Revista Mexicana de Ingeniería Química, 10(2): 281290 (2011) 
Wang, R., M. Zhang y A. S. Mujumdar, Effect of osmotic dehydration on microwave freeze-drying characteristics and quality of potato chips, Drying Technology, 28(6), 798-806 (2010)

Zapata, J. y A. Montoya, Deshidratación osmótica de láminas de mango cv. Tommy atkins aplicando metodología de superficies de respuesta. Universidad de Antioquia, Revista de Facultad de Ciencias Agrarias, 65(1), 6507-6518 (2012)

Zou, K., T. Jianwen, Li. Huang, D. Xinwei y W. Baoyao, Effect of osmotic pretreatment on quality of mango chips by explosion puffing drying. Food Science and Technology, 51, 253-259 (2013)

Zuluaga J., M. Cortes y E. Rodríguez, Evaluación de las características físicas de mango deshidratado aplicando secado por aire caliente y deshidratación osmótica. Universidad Nacional de Colombia, Medellín. Revista de la Facultad de Ingeniería, 25(4), 127-135 (2010)

Zuluaga, A., Diseño de metodologías para un equipo universal de ensayos con aplicación en la industria alimenticia, Universidad tecnológica de Pereira, Química industrial, Escuela de Tecnología Química, Pereira (2012) 